\title{
NITROFURANTOIN: THE TIME-TESTED CHOICE IN UNCOMPLICATED URINARY TRACT INFECTION
}

\author{
1Demonstrator, Department of Microbiology, NRS Medical College, Kolkata. \\ ${ }^{2}$ Assistant Professor, Department of Microbiology, NRS Medical College, Kolkata. \\ ${ }^{3}$ Assistant Professor, Department of Microbiology, NRS Medical College, Kolkata. \\ ${ }^{4}$ Assistant Professor, Department of Microbiology, NRS Medical College, Kolkata. \\ ${ }_{5}^{5}$ Demonstrator, Department of Microbiology, NRS Medical College, Kolkata. \\ ${ }^{6}$ Professor, Department of Microbiology, NRS Medical College, Kolkata.
}

Jayashree Konar ${ }^{1}$, Ranadeep Ghosh², Shiv Sekhar Chatterjee ${ }^{3}$, Amit Kumar Majumdar $^{4}$, Mrinmoy Pathak $^{5}$, Susmita Bhattacharya ${ }^{6}$

\begin{abstract}
Uropathogenic Escherichia Coli (UPEC) is the leading pathogen that causes community acquired as well as nosocomial uncomplicated urinary tract infection throughout the world. The study was conducted for one year with 2557 clinically suspected cases, both In-patients and Out-patients, to find out the most common pathogenic bacteria to cause uncomplicated urinary tract infection in a tertiary care hospital, also to determine the sensitivity pattern of the leading uropathogens against commonly prescribed antimicrobials in uncomplicated urinary tract infection. Significant bacteriuria was found in 811 cases (31.71\%) in this study. Amongst them, Escherichia coli was isolated in 335 (41.3\%) cases and female patients outnumbered the male patients (195, i.e. 58\%). Enterococcus spp. (110, i.e. 13.56\%) heads the list of Gram positive uropathogens (155 cases, i.e. 19.11\%). Candida spp. was isolated in $6.16 \%$ of total culture positive cases. Most of the isolated Escherichia coli were sensitive to nitrofurantoin (290, i.e. 87\%). Majority of the nitrofurantoin sensitive Escherichia coli was found to be resistant to other broad spectrum antimicrobials. Carbapenemase producers was found to be 220 (75.86\%) and 255 (87.93\%) were Extended Spectrum Beta Lactamase (ESBL) producers. Most (87.93\%) of the Escherichia coli isolates were resistant to Fluoroquinolones. Among nitrofurantoin resistant Escherichia coli, sensitivity to carbapenems was only $33.33 \%$. Thus Nitrofurantoin in Urinary Tract Infection may be used as an empirical drug even in the era of super bugs.
\end{abstract}

\section{KEYWORDS}

Urinary Tract Infection, Escherichia Coli, Nitrofurantoin.

HOW TO CITE THIS ARTICLE: Konar J, Ghosh R, Chatterjee SS, et al. Nitrofurantoin: the time-tested choice in uncomplicated urinary tract infection. J. Evolution Med. Dent. Sci. 2016;5(34):1872-1875, DOI: 10.14260/jemds/2016/442

\section{INTRODUCTION}

Urinary Tract Infection (UTI) is one of the most common health problems in the nosocomial as well as in the community setups. ${ }^{1}$ Uropathogenic Escherichia Coli (UPEC) are the leading pathogen in both community acquired and nosocomial UTI throughout the world. ${ }^{2}$ Uncomplicated infections occur most commonly in otherwise healthy women when uropathogenic bacteria, usually Escherichia coli, ascend from the perineum into the bladder and overcome host innate immunity. ${ }^{3}$ Complicated infections occur in patients with an anatomical or functional abnormality of the urinary tract. ${ }^{4}$ Nitrofurantoin is an age old cost effective therapeutic option for uncomplicated UTI. It is on the World Health Organization's List of Essential Medicines, the most important medications needed in a basic health system. ${ }^{5}$

Nitrofurantoin has been available for the treatment of UTIs since 1953. Its current uses include the treatment of uncomplicated UTIs and prophylaxis against UTIs in people prone to recurrent UTIs.6 The drug works by causing damage to the bacterial DNA, since its reduced form is highly reactive. This is made possible by the rapid reduction of nitrofurantoin inside the bacterial cell by flavoproteins (Nitrofuran reductase) to multiple reactive intermediates that attack ribosomal proteins, DNA, enzymes involved in respiration

Financial or Other, Competing Interest: None.

Submission 09-03-2016, Peer Review 04-04-2016,

Acceptance 11-04-2016, Published 26-04-2016.

Corresponding Author:

Dr. Susmita Bhattacharya,

Professor, Department of Microbiology,

Nil Ratan Sircar Medical College,

138 AJC Bose Road,

Kolkata-700014.

E-mail: subhatchaj@yahoo.co.in

DOI: $10.14260 /$ jemds/2016/442 and pyruvate metabolism within the cell.

Nitrofurantoin exerts greater effects on bacterial cells than mammalian cells as the former activate the drug more rapidly. Multiple mechanisms of action may likely be responsible for the low development of resistance against this drug. ${ }^{7}$

Advantages of nitrofurantoin include near absence of adverse effects even in pregnancy, as it belongs to pregnancy category B antimicrobials. ${ }^{8}$ A meta-analysis of four out of twenty-two available studies on the safety of nitrofurantoin has found no increased risk in pregnancy. ${ }^{9}$ Leading urinary pathogens are much more susceptible to this drug than other empirically used antibiotics like co-trimoxazole, fluoroquinolones and carbapenems. ${ }^{10}$ Nitrofurantoin, therefore, may be advocated as the drug of choice for prophylaxis as well as treatment of UTI even in this era of super bugs.

The diagnosis of UTI is made on the basis of symptoms and diagnostic precision is improved by urinalysis. Urine culture is important with severe, recurrent or complicated infection and also when the diagnosis is unclear - in children and the elderly. Most women with symptoms that resolve quickly do not require further investigation, but in children, men and patients with recurrent or severe infection imaging of the renal tract, functional testing and cystoscopy should be considered to exclude an underlying abnormality. ${ }^{11,12}$

Empirical antibiotic treatment may be started on the basis of symptoms for uncomplicated cystitis, but should be altered based on culture results for more severe infections. Antibiotic treatment is usually sufficient for uncomplicated cystitis in women. Long-term or post-coital antibiotics are advised for patients with recurrent infection in whom nonantibiotic strategies have failed.13,14 
Now-a-days, there are different guidelines that emphasize on the use of Fluoroquinolones and Cotrimoxazole. Again, Carbapenems in spite of being a parenteral option, are also preferred by several schools. ${ }^{15,16}$

Keeping these issues in mind, the present study was undertaken with a view to determine the leading uropathogen along with its antimicrobial susceptibility pattern in order to find out an effective prophylactic and therapeutic option for uncomplicated UTI.

\section{AIMS AND OBJECTIVES}

1. Identification of the most common pathogenic bacteria causing uncomplicated urinary tract infection in a tertiary care hospital in the last one year (2015).

2. Determine the sensitivity pattern of this leading uropathogen against commonly prescribed antimicrobials in uncomplicated urinary tract infection.

\section{MATERIAL AND METHODS}

The study was performed from January 2015 to December 2015 with 2557 clinically suspected patients of uncomplicated urinary tract infection. Symptomatic presence of any of the clinical symptoms of UTI like urinary urgency, hesitancy, increased frequency of micturition, pyrexia and dysuria were under the inclusion criteria. Immunocompromised patients, patients with renal transplantation and any other comorbidity were excluded from the study. Microbiological work-up to isolate and identify the bacterial pathogen was done as per standard protocol from aseptically collected urine according to standard guidelines. ${ }^{17}$ Antibiogram was done by Kirby Bauer disk diffusion technique and interpretation was done according to Clinical Laboratory Standard Institute (CLSI) guidelines. ${ }^{17}$ Data compilation and interpretation was performed as per standard statistical methods. For antibiotic susceptibility testing, drug discs of Nitrofurantoin $(300 \mu \mathrm{g})$, Imipenem $(10 \mu \mathrm{g})$, Meropenem $(10 \mu \mathrm{g})$ Levofloxacin $(5 \mu \mathrm{g})$, Ceftazidime $(30 \mu \mathrm{g})$, Ceftazidime/clavulanic acid $(30 / 10 \mu \mathrm{g})$ and Amikacin $(30 \mu \mathrm{g})$ were used.

Phenotypic Confirmatory Test for Detection of Extended Spectrum Beta Lactamase (Combination Disc Diffusion Test as Per CLSI Guidelines). ${ }^{17}$

Ceftazidime $(30 \mu \mathrm{g})$ disc alone and Ceftazidime/clavulanic acid $(30 \mu \mathrm{g} / 10 \mu \mathrm{g})$ were placed at a distance of $25 \mathrm{~mm}$, centre to centre, on a Mueller Hinton Agar plate inoculated with a bacterial suspension of $0.5 \mathrm{McF}$ arland turbidity. Overnight incubation then done at $37^{\circ} \mathrm{C}$.

If the zone of inhibition is $\geq 5 \mathrm{~mm}$ diameter for a combination disc versus Ceftazidime disc alone then the strain is confirmed as ESBL producer by phenotypic method.

\section{Tests for Carbapenemase Producer Detection. ${ }^{17}$}

Bacterial isolates producing $\leq 13 \mathrm{~mm}$ zone of inhibition around the Imipenem and Meropenem discs by Kirby Bauer disc diffusion test were screened for carbapenemase detection and phenotypic confirmation was done by Modified Hodge Test; 0.5 McFarland dilution of the Escherichia coli ATCC 25922 in $5 \mathrm{~mL}$ of broth was prepared. A 1:10 dilution of this strain was cultured by lawn technique on Mueller Hinton agar plate. A $10 \mu \mathrm{g}$ meropenem disc was placed in the centre of the test area. Test organism was streaked in straight line from the edge of the disc to the edge of the plate. The plate was then incubated overnight at $37^{\circ} \mathrm{C}$ in ambient air. A positive test produced a clover leaf-like indentation of the Escherichia coli 25922, whereas a negative test showed no indentation or growth of the Escherichia coli 25922 within the zone of inhibition.

\section{RESULT}

The study was conducted for a period of one year with 2557 clinically suspected cases, both In-patients and Out-patients. A fair proportion of the patients gave the history of catheterization or instrumentation. It was found that significant number of bacteria could be isolated in 811 cases (31.71\%). Amongst them Escherichia coli were isolated in 335 cases (41.3\%), whereas Gram positive uropathogen was isolated in 155 cases (19.11\%). Enterococcus spp. headed the list of Gram positive uropathogens - 110 cases (13.56\%). Candida spp. was isolated in 6.16\% (Table 1).

Majority (195 cases - 58\%) of isolated Escherichia coli was reported from female cases (Figure 1).

Most of the isolated Escherichia coli were sensitive to nitrofurantoin (290 isolates - 87\%) (Figure 2) and the rest 45 $(13.43 \%)$ isolates were found to be resistant to this drug.

Majority of the nitrofurantoin sensitive Escherichia coli was resistant to other broad spectrum antimicrobials. Amongst them, 220 (75.86\%) were carbapenemase producers, 255 (87.93\%) were Extended Spectrum Beta Lactamase (ESBL) producers and 170 (58.62\%) were resistant to aminoglycoside antimicrobials. Most (87.93\%) of the Escherichia coli isolates were resistant to fluoroquinolone antibiotics (Figure 3).

In $33.33 \%$ cases, uropathogenic Escherichia coli were found to be sensitive to carbapenems, although they were resistant to nitrofurantoin (Figure 4).

\begin{tabular}{|c|c|}
\hline $\begin{array}{l}\text { Name of the } \\
\text { Uropathogen }\end{array}$ & $\begin{array}{l}\text { Number of Isolates } \\
\quad(\text { Total }=811)\end{array}$ \\
\hline Escherichia coli & $335(41.3 \%)$ \\
\hline Klebsiella pneumoniae & $134(16.52 \%)$ \\
\hline Citrobacter spp. & $35(4.31 \%)$ \\
\hline Proteus mirabilis & $19(2.34 \%)$ \\
\hline Providencia spp. & $8(0.98 \%)$ \\
\hline Morganella morganii & $5(0.62 \%)$ \\
\hline Acinetobacter spp. & $35(4.31 \%)$ \\
\hline Pseudomonas aeruginosa & $35(4.31 \%)$ \\
\hline Staphylococcus aureus & $45(5.54 \%)$ \\
\hline Enterococcus spp. & $110(13.56 \%)$ \\
\hline Candida spp. & $50(6.16 \%)$ \\
\hline \multicolumn{2}{|c|}{$\begin{array}{l}\text { Table 1: Distribution of Different Cultivable Uropathogens } \\
\text { from January } 2015 \text { to December } 2015(n=811)\end{array}$} \\
\hline
\end{tabular}

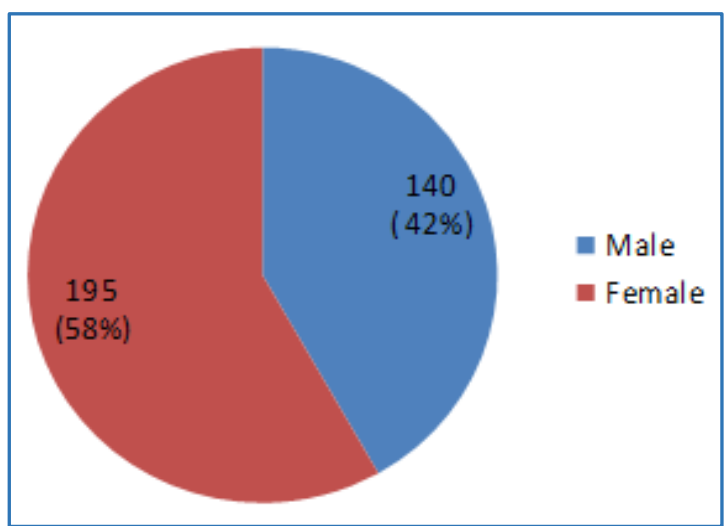

Fig. 1: Pie Chart showing Gender Distribution of Uropathogenic Escherichia coli $(n=335)$ 


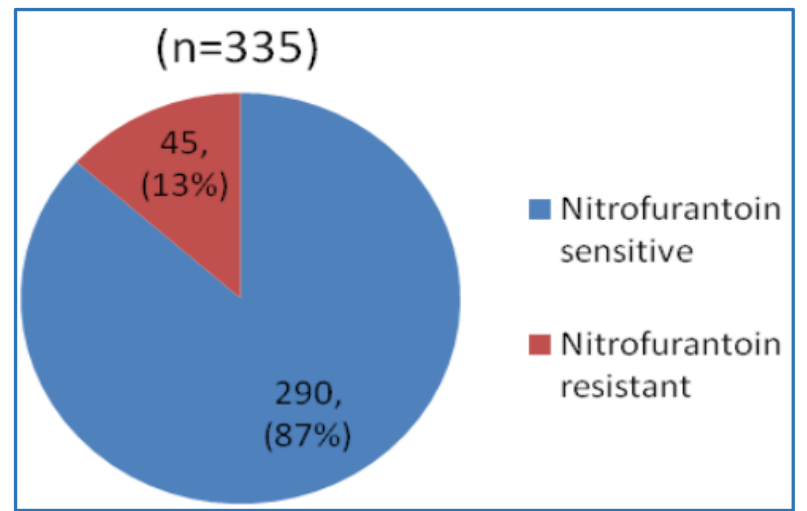

Fig. 2: Pie Chart showing Sensitivity Pattern of Nitrofurantoin on Uropathogenic Escherichia coli

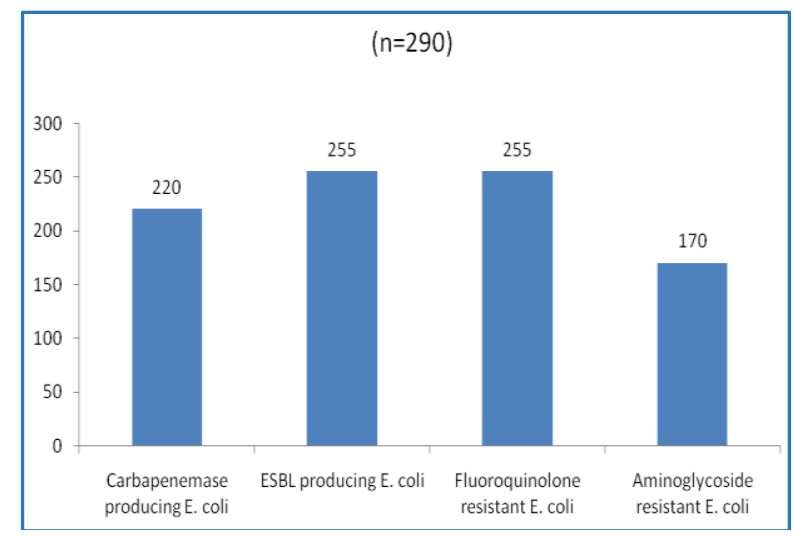

Fig. 3: Bar Diagram showing Resistance Pattern of Nitrofurantoin Sensitive Uropathogenic Escherichia coli Isolates

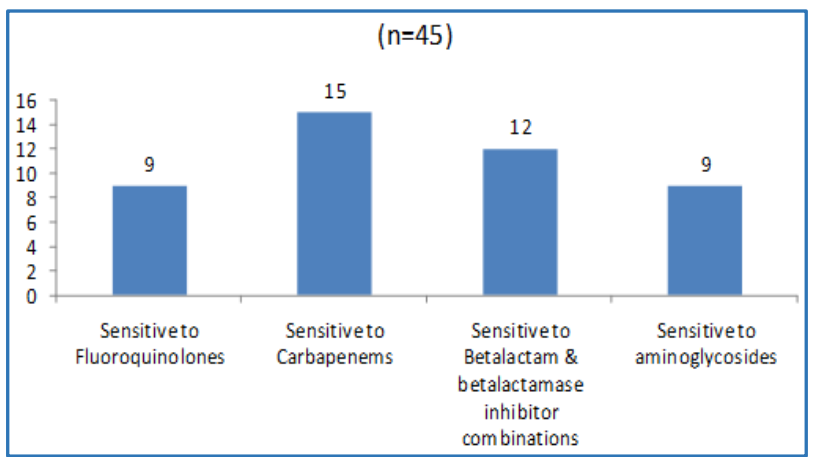

Fig. 4: Bar Diagram showing Sensitivity Pattern of Nitrofurantoin Resistant Uropathogenic Escherichia coli Isolates

\section{DISCUSSION}

The study was conducted for a period of one year with 2557 clinically suspected UTI cases, both In-patients and Outpatients. A fair proportion of the patients gave history of catheterization or instrumentation. It was found that significant number of bacteria could be isolated in 811 cases (31.71\%). The culture negative cases may be caused by fastidious pathogens or may be because of institution of empirical antimicrobials before sample collection. Majority (195, i.e. 58\%) of isolated Escherichia coli was reported from female cases. Amin et al found 553 (8.7\%) patients to be urine culture positive ( $68 \%$ females) in their study conducted in Iran. 18

Amongst these 811 culture-positive cases, Escherichia coli was isolated in 335 cases (41.3\%) when the total number of Gram negative pathogens was 606. The Gram positive uropathogens were isolated in 155 cases $(19.11 \%)$ out of the total number of 811 culture positive cases. Enterococcus spp. headed the list of Gram positive uropathogens (110 out of 811 cases, i.e. $13.56 \%)$. Candida spp. was isolated in 50 cases, i.e. $6.16 \%$ of total culture positive cases. Study conducted by Komala et al in India showed distribution of various microbes for causing the UTI: E. coli $79 \%$, S. saprophyticus $11 \%$, Klebsiella 3\%, Proteus spp. 2\%, Enterococcus 2\%, others 2\%, thus E. coli being the leading uropathogen. ${ }^{19}$

Most (290, i.e. 87\%) of the isolated Escherichia coli were sensitive to nitrofurantoin. Similarly, R Nalini et al in India found that $85.19 \%$ UPEC were sensitive to nitrofurantoin and Kibret $\mathrm{M}$ et al from Ethiopia reported $96.4 \%$ of UPEC isolates to be sensitive to nitrofurantoin. ${ }^{20,21}$ Majority of the 290 nitrofurantoin sensitive Escherichia coli were resistant to other broad spectrum antimicrobials: 220 (75.86\%) were carbapenemase producers, 255 (87.93\%) were Extended Spectrum Beta Lactamase (ESBL) producers, $170(58.62 \%)$ were resistant to aminoglycoside antimicrobials and $255(87.93 \%)$ were resistant to fluoroquinolone group of antibiotics. According to the study conducted by Zhanel G et al, Mean Resistance Rates for 2,000 urinary tract isolates collected from outpatients across Canada in 1998 for ampicillin, trimethoprimsulfamethoxazole, mecillinam, nitrofurantoin and ciprofloxacin were 41.1, 19.2, 14.7, 5.0 and 1.8\%, respectively. For Escherichia coli isolates alone $(n=1,681)$ comparable rates were $41.0,18.9,7.4,0.1$ and $1.2 \%$, respectively. ${ }^{22}$ However, Goenka et al showed that among the tested antibiotics, the susceptibility of Gram negative bacteria against piperacillin-tazobactam, imipenem, amikacin and gentamicin. ${ }^{23}$ was found to be quite high.

Several workers like Kromann-Andersen B et al, has concluded that ofloxacin is an orally active drug, which offered a valuable alternative to other broad spectrum antibacterial drugs. ${ }^{24}$ In the present study, however, nitrofurantoin resistant UPEC were found to be sensitive to ofloxacin in only $20 \%$ cases. We have found that nitrofurantoin resistant uropathogens were mostly sensitive to carbapenems $(33.3 \%)$, followed by betalactam and betalactamase inhibitor combinations $(26.67 \%)$. This study also revealed that these strains were least sensitive to aminoglycosides and fluoroquinolones (20\% each). It may be due to injudicious use of these drugs in this particular setup.

\section{CONCLUSION}

UTI, one of the major health problems in India especially for female, is predominantly caused by Escherichia coli. Nitrofurantoin, a safe antimicrobial drug as per literature is found to be the most effective therapeutic option for treating uncomplicated UTI even in multidrug resistant UPEC. Thus, the use of nitrofurantoin in UTI may be advocated as an empirical drug even in the era of super bugs in our institution as revealed in this current study. Similar study should be continued to institute proper empirical therapy of the patients, because of the ever changing sensitivity pattern.

\section{LIMITATION}

The mechanism of drug resistance has not been studied in this current work. The strains have been preserved for future 
molecular characterization and determination of the cause of drug resistance.

\section{ACKNOWLEDGEMENTS}

We are very much grateful to the Principal, Dean - Student Affairs and Head of the Department of Microbiology, Nilratan Sircar Medical College and Hospital, Kolkata, for their kind permission and cooperation in conducting this study.

\section{REFERENCES}

1. Jellheden B, Norrby RS, Sandberg T. Symptomatic urinary tract infection in women in primary health care bacteriological, clinical and diagnostic aspects in relation to host response to infection. Scand J Prim Health Care 1996;14(2):122-8.

2. Uropathogenicescherichia coli: The pre-eminent urinary tract infection pathogen. Nova publishers. Retrieved 27 November, 2013.

3. Hooton TM, Scholes D, Hughes JP, et al. A prospective study of risk factors for symptomatic urinary tract infection in young women. N Engl J Med 1996;335:46874.

4. Scholes D, Hooton TM, Roberts PL, et al. Risk factors for recurrent urinary tract infection in young women. J Infect Dis 2000;182(4):1177-82.

5. WHO model list of essentialmedicines (PDF). World Health Organization. October 2013. Retrieved 22April,2014.

6. Garau J. Other antimicrobials of interest in the era of extended-spectrum beta-lactamases: fosfomycin, nitrofurantoin and tigecycline. Clin Microbiol Infect 2008;14(1):198-202.

7. Tu Y, McCalla DR. Effect of activated nitrofurans on DNA. Biochem Biophys Acta 1975;402(2):142-9.

8. Lee $M$, Bozzo $P$, Einarson $A$, et al. Urinary tract infections in pregnancy. Can Fam Physician 2008;54(6):853-4.

9. Ben David S, Einarson T, Ben David Y, et al. The safety of nitrofurantoin during the first trimester of pregnancy: meta-analysis. Fundam \& clinpharmacol 1995;9(5):5037.

10. Schultz HJ, Edson RS. Cystitis treatment in women, circa 2011: new role for an old drug. Mayo Clin Proc 2011;86(6):477-9.

11. Lin K, Fajardo K. Screening for asymptomatic bacteriuria in adults: evidence for the U.S. preventive services task force reaffirmation recommendation statement. Ann Intern Med 2008;149(1):W20-4.

12. Little $\mathrm{P}$, Turner $\mathrm{S}$, Rumsby $\mathrm{K}$, et al. Dipsticks and diagnostic algorithms in urinary tract infection: development and validation, randomised trial, economic analysis, observational cohort and qualitative study. Health Technol Assess 2009;13(9):iii-iv, ix-xi,173.
13. Grabe M, Bishop MC, Bjerklund-Johansen TE, et al Guidelines on urological infections. European Association of Urology, 2013.

14. Katchman EA, Milo G, Paul M, et al. Three-day vs longer duration of antibiotic treatment for cystitis in women: systematic review and meta-analysis. Am J Med 2005;118(11):1196-207.

15. McCarty JM, Richard G, Huck W, et al. A randomized trial of short-course ciprofloxacin, ofloxacin, or trimethoprim/sulfamethoxazole for the treatment of acute urinary tract infection in women ciprofloxacin urinary tract infection group. Am J Med 1999;106(3):292-9.

16. Barber AE, Norton JP, Spivak AM, et al. Urinary tract infection: current and emerging management strategies. Clin Infect Dis 2013;57(5):719-24.

17. Clinical and laboratory standards institute (CLSI; formerly NCCLS). Urinalysis and collection, transportation, and preservation of urine specimens; approved guideline- Second Edition. Document GP16A2. Wayne PA, 2001;21(19).

18. Mansour Amin, Manijeh Mehdinejad, Zohreh Pourdangchi. Study of bacteria isolated from urinary tract infections and determination of their susceptibility to antibiotics. Jundishapur Journal of Microbiology 2009;2(3):118-23.

19. Komala M, Sampath Kumar. Urinary tract infection: causes, symptoms, diagnosis and it's management. Indian Journal of Research in Pharmacy and Biotechnology 2013;1(2):226-33.

20. Nalini R, Ezhil Ramy J, Meenakshi B, et al. Recent sensitivity pattern of escherichia coli in urinary tract infection. RRJMB 2014;3(3):31-35.

21. Kibret M, Abera B. Antimicrobial susceptibility patterns of E coli from clinical sources in northeast Ethiopia. African Health Sciences 2011;11(1):S40-5.

22. Zhanel G, Karlowsky J, Harding G, et al. A canadian national surveillance study of urinary tract isolates from outpatients: comparison of the activities of trimethoprim- sulfamethoxazole, ampicillin, mecillinam, nitrofurantoin, and ciprofloxacin. Antimicrob Agents Chemother 2000;44(4):1089-92.

23. Abhishek S Goenka, Rajesh P Karyakarte, Sumit S Aggarwal, et al. Study of bacteriological profile of urinary tract infection among patient attending tertiary care center. International Journal of Information Research and Review 2015;2(7):929-32.

24. Kromann-Andersen B, Kroyer Nielsen K. Ofloxacin in urinary tract infections. Scand J Infect Dis Suppl 1990;68:35-40. 